\title{
Simultaneous estimation of serum and cerebrospinal fluid adenosine deaminase level to differentiate tuberculous and non-tuberculous meningitis
}

\author{
Shrivastava $\mathbf{J}^{1}$, Agrawal $\mathbf{A}^{2}$, Palel $\mathbf{D}^{3}$ \\ ${ }^{1}$ Dr Jyotsna Shrivastava, Professor, Department of Paediatrics, Gandhi Medical College \& Kamla Nehru Hospital, Bhopal, \\ MP, India, ${ }^{2}$ Dr Amit Agrawal, Assistant Professor, Department of Paediatrics, Gandhi Medical College \& Kamla Nehru \\ Hospital, Bhopal, MP, India, ${ }^{3}$ Dr Dharmes Patel, Consulting Pediatrician, Bhopal, MP, India
}

Address for correspondence: Dr Amit Agrawal, Email: agrawaldramit@yahoo.co.in

\begin{abstract}
Introduction: Studies have shown the role of cerebrospinal fluid (CSF) adenosine deaminase (ADA) level but simultaneously estimation of serum and CSF-ADA to diagnose tuberculous meningitis (TBM) has been assessed rarely. Therefore, we conducted this study to assess the usefulness of serum and CSF-ADA in diagnosing TBM and to differentiate it with non-tuberculous meningitis in children. Material \& Methods: A prospective, case-control study was conducted in tertiary care institution of central India over one year. Children hospitalised with suspected meningitis were recruited and serum and CSF-ADA levels were estimated after dividing them into TBM and non-tuberculous meningitis. Results: Out of 78 recruited cases, 36 had TBM while 42 had non-tuberculous etiology. 32 of 36 TBM cases had CSF-ADA above cut-off value (10U/L) while only 3 out of 42 controls had levels above this. Serum ADA above cut-off (60U/L) was seen in 13 TBM and 2 non-TBM cases. Mean CSF-ADA in cases (18.68 $\pm 6.21 \mathrm{U} / \mathrm{L})$ was significantly higher than in controls $(5.98 \pm 2.75 \mathrm{U} / \mathrm{L})(\mathrm{p}<0.0001)$; however, serum ADA did not differ significantly between two groups ( $\mathrm{p}=0.0631)$. CSF-ADA $>10 \mathrm{U} / \mathrm{L}$ showed $92.86 \%$ sensitivity and $88.89 \%$ specificity (area under ROC curve -0.9742 ) in differentiating tuberculous from non-tuberculous meningitis; it also has $91.43 \%$ positive predictive value and $90.70 \%$ negative predictive value. Serum ADA $>60 \mathrm{IU} / \mathrm{L}$ has shown sensitivity of $95.24 \%$ with poor specificity of $33.33 \%$. Conclusion: CSF-ADA estimation is of considerable value to diagnose TBM and to differentiate it from non-tuberculous meningitis but simultaneous estimation of CSF and serum ADA has no additional benefit.
\end{abstract}

Key words: Tuberculous meningitis, Adenosine deaminase, CSF, Serum, Children

\section{Introduction}

Tuberculosis is a chronic infectious disease appropriately referred to as "Captain of ship of Death", which can involve almost every organ of the body. Of these, neurotuberculosis is the most severe form of tuberculosis, and it still remains an important cause of morbidity and mortality despite availability of the effective treatment [1]. This is mainly due to the lack of a single sensitive and specific diagnostic test, which can detect tuberculous meningitis (TBM) at an early stage. Definitive diagnosis of is established by observation of acid fast bacilli in Ehrlich-Ziehl-Neelsen (EZN) stains of cerebrospinal fluid (CSF) and/or isolation of mycobacterium in CSF culture, which remains the gold standard to diagnose tuberculosis. Unfortunately, these tests are not very sensitive and the conventional culture methods take a long time (upto 4-6 weeks) to give results [2-4].

Manuscript received: $4^{\text {st }}$ May 2014

Reviewed: $10^{\text {th }}$ May 2014

Author Corrected: $16^{\text {th }}$ May 2014

Accepted for Publication: $21^{\text {st }}$ May 2014
Outcome in TBM is strongly associated with the stage of disease at presentation. Therefore, early diagnosis and treatment of this disease can reduce mortality and sequel rates considerably. Studies are required to develop diagnostic methods, which can detect tuberculosis early and should be sensitive as well as specific enough. Adenosine deaminase (ADA) is an enzyme involved in purine catabolism leading to hydrolytic deamination of adenosine to inosine and ammonia secreted by monocytes, macrophages and $\mathrm{T}$ lymphocytes. Its production occurs to a greater extent by more differentiated or activated T-lymphocytes and an increase in plasma ADA levels is observed in diseases where the cellular immunity response is actively involved e.g. tuberculosis [3-4].

Although, many studies have reported elevated CSFADA levels in TBM [2,5-6], very few studied the role of simultaneous estimation of serum and CSF-ADA levels,

Available online at: $\underline{\text { www.ijmrr.in }} 414$ | P a g e 
especially in children [7-8]. Therefore, we conducted this prospective study with an aim to determine the usefulness of CSF and serum ADA levels to diagnose TBM and to differentiate it from non-tuberculous meningitis.

\section{Material \& Methods}

This prospective study was conducted in the pediatrics department of a tertiary care teaching institution of central India over a period of 12 months. Clearance from the institutional ethics committee was obtained and written informed consent from the parents or legal guardians was obtained before recruitment. Patients admitted to pediatric ward with signs and symptoms of meningitis were included in the study and were divided into tuberculous and non-tuberculous meningitis according to the accepted criteria. Patients already receiving anti-tubercular therapy, patients with known diagnosis or having being readmitted were excluded from the study.

Patient's demographic details were collected in a predesigned proforma and details of thorough history and examination findings were also entered. Venous blood sample was collected by peripheral venepuncture and sent for routine blood investigations as well as to estimate serum ADA levels.

Lumbar puncture was done under all aseptic precautions in each case to collect at least $2 \mathrm{ml}$ of CSF in a sterile vial and was sent to laboratory for biochemical and microscopic examination by the personnel unaware of clinical diagnosis. Hemorrhagic CSF was excluded from the study. Both CSF and serum ADA activity was estimated in all these patients by the calorimetric method of Galanti and Guisti [18] and was expressed as U/L.
Confirmed or probable TBM patients were taken as cases while the patients having features of meningitis but not fulfilling above mentioned criteria were taken as controls, which included patients with pyogenic meningitis, viral meningo-encephalitis, and cerebral malaria. TBM was confirmed by demonstration of acid fast bacilli in the CSF. Probable disease was diagnosed in the presence of essential plus two or more of the supportive criteria:

Essential criteria: CSF WBC counts $>100 / \mathrm{mm} 3$ and lymphocytic pleocytosis $(>60 \%$ ), CSF protein $>75$ $\mathrm{mg} / \mathrm{dL}$ and low CSF glucose $(<60 \%$ of matched plasma glucose).

Supportive findings: Subacute or chronic outset and slow clinical progression, history of contact with tuberculosis, positive skin reaction $(>10 \mathrm{~mm}$ induration to 5 tuberculin unit purified protein derivative), generalized lymphadenopathy or histologically proven tuberculous lymphadenitis, positive radiological evidence of tuberculosis elsewhere in the body, CT scan evidence of basal exudates or CNS tuberculosis, isolation of AFB from gastric lavage or other sites.

Results for the baseline characteristics and clinical data were described as mean $\pm \mathrm{SD}$ with range and were compared between groups by independent t-test. To determine differences between groups in terms of ADA values, Mann-Whitney test was done.

ROC curve analysis was performed to determine the cutoff value for ADA to differentiate TBM and non-TBM groups. Statistical analysis was performed by SPSS (version 16.0) package program. Significance level was accepted as $5 \%$.

\section{Results}

During the study period, total 78 cases were recruited with median age of 4.7 years (range 4 months to 12 years) and male: female ratio of 2.3:1. Of these, 36 cases fulfilled the criteria to be diagnosed as TBM while remaining 42 cases had nontuberculous meningitis $\{18$ - pyogenic meningitis (PM), 15 - viral meningo-encephalitis (VM), and 9 - cerebral malaria $(\mathrm{CM})\}$. Only one $(2.78 \%)$ patient in 36 cases with TBM had evidence of presence of Mycobacterium.

On taking $10 \mathrm{IU} / \mathrm{L}$ as cut-off value for CSF-ADA, 32 of the 36 tuberculous patients had values above the cut-off value, as against only 3 out of 42 non-tuberculous patients had high CSF-ADA values. Similarly, serum ADA was elevated in 13 cases with TBM and 2 non-tuberculous patients above the cut-off value (60 IU/L) as shown in table 1.

Mean CSF-ADA was significantly $(\mathrm{p}>0.0001)$ higher in patients with TBM $(18.68 \pm 6.21,95 \%$ CI $-16.65-20.71)$ than those with non-tuberculous meningitis (5.98 $\pm 2.75,95 \% \mathrm{CI}-5.15-6.81)$. However, serum ADA level showed only non-significant (0.0631) elevation in TBM patients $(51.41 \pm 20.52,95 \% \mathrm{CI}-44.71-58.11)$ in comparison to patients with non- 
Table 1 - Number of patients with elevated CSF and Serum ADA levels

\begin{tabular}{|l|l|l|l|l|l|}
\hline Group & \multirow{2}{*}{ Total no. of cases } & \multicolumn{2}{l|}{ CSF ADA } & \multicolumn{2}{l|}{ Serum ADA } \\
\cline { 3 - 6 } & & Raised & \% & \multicolumn{2}{l|}{ Raised } \\
\hline Tuberculous Meningitis & 36 & 32 & 88.89 & 13 & 36.11 \\
\hline Pyogenic Meningitis & 18 & 2 & 11.11 & 2 & 11.11 \\
\hline Viral Meningo-encephalitis & 15 & 1 & 6.67 & 0 & 0 \\
\hline Cerebral Malaria & 9 & 0 & 0 & 0 & 0 \\
\hline Total & 78 & 35 & 44.49 & 15 & 19.23 \\
\hline
\end{tabular}

tuberculous meningitis (41.55 $12.02,95 \%$ CI - 37.91-45.19). Mean values for CSF and serum ADA in cases (TBM) and different groups of non-tuberculous patients (PM, VM and CM) are shown in table 2. CSF-ADA in TBM cases were significantly high in comparison to other three groups of non-TBM ( $p<0.0001)$. However, there was no significant difference was seen in serum ADA between TBM group and 3 groups of non-tuberculous meningitis patients separately (table 2).

Table 2 - Serum and CSF ADA levels in different groups of patients

\begin{tabular}{|c|c|c|c|c|c|}
\hline Patients & $\mathbf{n}$ & $\begin{array}{l}\text { Range } \\
\text { (Median) }\end{array}$ & Mean \pm SD & $P$ - value & 95\% Confidence Interval \\
\hline \multicolumn{6}{|l|}{ Serum ADA levels (in U/L) } \\
\hline Tuberculous Meningitis & 36 & $20.2-108(45.7)$ & $51.41 \pm 20.52$ & & $44.71-58.11$ \\
\hline Pyogenic Meningitis & 18 & $\begin{array}{l}24-68.2 \\
(44.1)\end{array}$ & $44.12 \pm 12.64$ & 0.2677 & $38.29-49.97$ \\
\hline Viral Meningo-encephalitis & 15 & $\begin{array}{l}13.6-55.8 \\
(43.6)\end{array}$ & $40.73 \pm 11.50$ & 0.1508 & $34.91-46.55$ \\
\hline Cerebral Malaria & 9 & $\begin{array}{l}12.7-51.8 \\
(40.4)\end{array}$ & $37.83 \pm 12.53$ & 0.1251 & $29.64-46.02$ \\
\hline \multicolumn{6}{|l|}{ CSF ADA levels (in U/L) } \\
\hline Tuberculous Meningitis & 36 & $\begin{array}{l}6.2-33.4 \\
(18.05)\end{array}$ & $18.68 \pm 6.21$ & & $16.65-20.71$ \\
\hline Pyogenic Meningitis & 18 & $\begin{array}{l}3.1-14.5 \\
(6.1)\end{array}$ & $6.82 \pm 3.27$ & $<0.0001$ & $5.31-8.33$ \\
\hline Viral Meningo-encephalitis & 15 & $\begin{array}{l}1.3-11.5 \\
(5.5)\end{array}$ & $5.93 \pm 2.39$ & $<0.0001$ & $4.72-7.14$ \\
\hline Cerebral Malaria & 9 & $\begin{array}{l}2.2-6.1 \\
(4.1)\end{array}$ & $4.41 \pm 1.32$ & $<0.0001$ & $3.55-5.27$ \\
\hline
\end{tabular}

Following receiver-operating characteristic curves (ROC) analysis for ADA activity in TBM and non-TBM groups, and with a cut-off value $>10 \mathrm{IU} / \mathrm{L}$ for CSF-ADA activity, it has $92.86 \%(95 \% \mathrm{CI}-80.52 \%-98.50 \%)$ sensitivity and $88.89 \%$ (95\% CI - 73.94\%-96.89\%) specificity for diagnosis of TBM. The area under the ROC curve (AUC) was $0.9742(95 \%$ CI - 0.9458 to 1.003 ) (fig. 1).

CSF-ADA level has positive predictive value $91.43 \%$ and negative predictive value $90.70 \%$. Serum ADA has good sensitivity of $95.24 \%$ (95\% CI - 83.84\%-99.42\%) for diagnosis of TBM on taking cut-off value at 60 IU/L but specificity reduced to $33.33 \%$ (95\% CI - 18.56\%-50.97\%) only. The area under the ROC curve (AUC) was 0.6227 (95\% CI - 0.4905 to 0.7549 ) (fig. 2). Serum ADA has a positive predictive value of $87.50 \%$ and negative predictive value only $64.52 \%$.

\section{Discussion}

There is considerable urgency in establishing the correct diagnosis of tuberculosis in patients with meningitis as treatment is most effective when instituted early in the course of the illness and delayed diagnosis may be associated with many serious CNS complications. The definitive diagnosis of TBM can be made by demonstration of tubercle bacilli in 
ROC curve for CSF-ADA Level

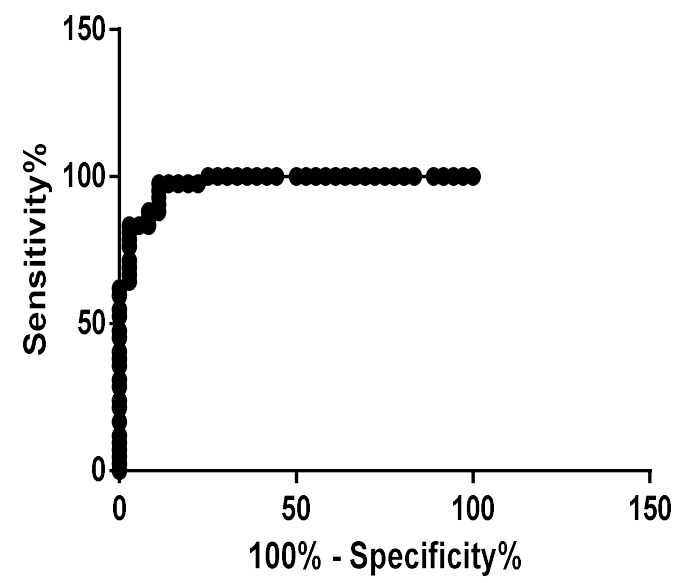

Fig 1: Receiver Operating Curve for CSF-ADA level (AUC - 0.9742)

CSF either by smear and/or culture. However, direct CSF smear methods are often negative and culture confirmation is positive only in $25-75 \%$ of cases with a delay of 4-6 weeks to show the growth [5-7]. Newer diagnostic methods e.g. DNA-PCR and various immunoassays (to detect antigens and/or antibodies in the CSF) are also not reliable due to variable sensitivity and specificity, insufficient available data as well as limited availability in the developing countries [8-13]. Hence, despite extensive work on TBM, a simple, rapid and sensitive test to establish an early diagnosis is still needed.

ADA has been considered as a marker of cell-mediated immunity. As both humoral and cell-mediated immunity play an important role in TBM, it has been suggested that estimation of CSF-ADA levels may help differentiate TBM from other infectious meningitis or non-infectious neurological disorders [14-19].

However, cut-off values of CSF-ADA activity and their sensitivity and specificity rates varied widely in different reports. Recently, authors of a meta-analysis evaluating ten studies reported that cut-off values for CSF-ADA in TBM were variable between $8.5-15.5$ with sensitivity varying between $50-100 \%$ and specificity between 60 $100 \%$ [20].

Different values for CSF-ADA in various studies may due to be study method, gender, age and race factors as well as the disease stage during which ADA is determined. Therefore, multicentre studies in different populations are needed to determine the standard CSFADA levels.

\section{ROC curve for Serum ADA levels}

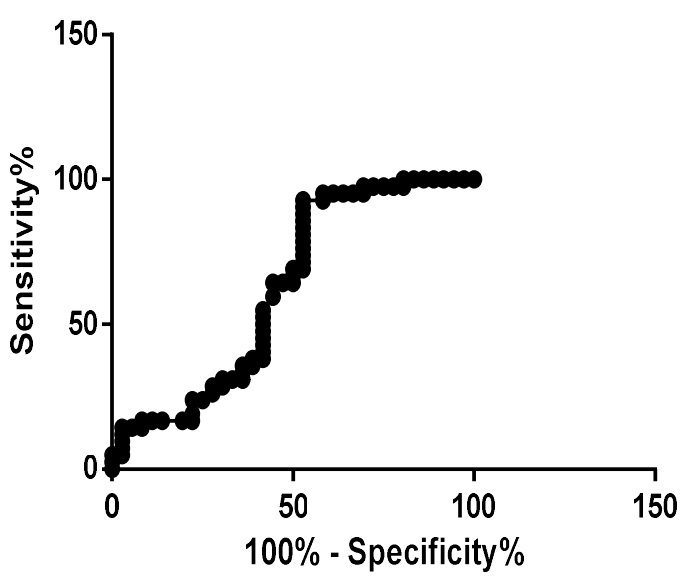

Fig 2: Receiver Operating Curve for Serum ADA level (AUC - 0.6227)

In our study, sensitivity of the CSF-ADA test was $92.86 \%$ and specificity was $88.89 \%$ on accepting the cutoff value of CSF-ADA activity as $10.0 \mathrm{IU} / \mathrm{L}$ for TBM and non-TBM differentiation. On the other hand, serum ADA showed sensitivity of $95.24 \%$ for diagnosis of TBM but specificity reduced to $33.33 \%$ on accepting the cut-off value as $60.0 \mathrm{IU} / \mathrm{L}$. only few studies evaluated the role of simultaneous estimation of serum and CSF-ADA for the diagnosis of TBM with conflicting results [7-8].

Our study results are supported by the study conducted by Donald et al [7], where they found raised mean values of CSF-ADA and CSF/plasma ADA ratio in TBM (12.61 $\mathrm{U} / \mathrm{L}$ and 0.24 respectively) and bacterial meningitis (15.43 U/L and 0.59 respectively) both. Both values were able to distinguish TBM from aseptic meningitis, but showed no added advantage over the CSF-ADA alone in the diagnosis of TBM.

On the contrary, Chaturvedi et al [8] reported that the specificity and sensitivity for CSF-ADA were 86 and $87 \%$, respectively on using the CSF-ADA value of 17.06 IU/L as cut-off value while specificity and sensitivity for serum ADA were 83 and $96 \%$ respectively on using a cutoff value $31.23 \mathrm{IU} / \mathrm{L}$.

They have suggested that simultaneous estimation of serum and CSF-ADA have an added advantage to differentiate between TBM and pyogenic meiningitis. Another recently conducted study involving adult patients showed increased specificity (from 92\% to 97\%) of combined serum and CSF ADA values in comparison to CSF-ADA alone. These different results can due to be 
the different study method, age and the disease stage during which ADA was determined.

\section{Conclusion}

Measurement of CSF-ADA is a simple, cost-effective and reliable biochemical test to differentiate TBM from non-tuberculous meningitis. On the other hand, simultaneous estimation of serum and CSF ADA measurement has no added advantage over CSF-ADA alone.

Thus, CSF-ADA estimation can be a useful tool to diagnose TBM cases especially in resource-limited countries like India where the incidence of tuberculosis is high and availability of other diagnostic tests for tuberculous meningitis is limited.

Author's contribution: DP: Acquisition and interpretation of data, data analysis, drafting the article, and literature review $\mathbf{A A}$ : Interpretation of data, data analysis, manuscript review, manuscript editing JS: Concept, drafting the article and literature review; revising the article critically for important intellectual content; JS will act as guarantor. All the authors approved the final manuscript.

\section{Funding: Nil}

Conflict of interest: None stated

Permission from IRB: Yes

\section{References}

1. Figaji AA, Fieggen AG. The neurosurgical and acute care management of tuberculous meningitis: Evidence and current practice. Tuberculosis (Edinb). 2010 Nov;90(6):393-400.

2. Tuon FF, Higashino HR, Lopes I, Litvoc MN, Atomiya AN, Antonangelo L, et al. Adenosine Deaminase and Tuberculous Meningitis - A Systematic Review with Meta-Analysis. Scand J Infect Dis. 2010 Mar;42(3):198207.

3. Molavi A, LeFrock JL. Tuberculous meningitis. Med Clin North Am. 1985 Mar;69(2):315-31.

4. Thwaites G, Chau TT, Mai NT, Drobniewski F, McAdam K, Farrar J: Tuberculous meningitis. J Neurol Neurosurg Psychiatry. 2000 Mar;68(3):289-99.

5. Moghtaderi A, Niazi A, Alavi-Naini R, Yaghoobi S, Narouie B. Comparative analysis of cerebrospinal fluid adenosine deaminase in tuberculous and non-tuberculous

\section{Research Article}

meningitis. Clin Neurol Neurosurg. 2010 Jul;112(6):459-62.

6. Choi SH, Kim YS, Bae IG, Chung JW, Lee MS, Kang $\mathrm{JM}$, et al. The possible role of cerebrospinal fluid adenosine deaminase activity in the diagnosis of tuberculous meningitis in adults. Clin Neurol Neuros. 2002 Jan;104(1):10-5.

7. Donald PR, Malan C, vander Walt A, Schoeman JF. The simultaneous determination of cerebrospinal fluid and plasma adenosine deaminase activity as a diagnostic aid in tuberculous meningitis. S Afr Med J. 1986 Apr $12 ; 69(8): 505-7$

8. Chaturvedi P, Vaidya J, Harinath BC, Pramanick B. Adenosine deaminase levels in cerebrospinal fluid and serum in the diagnosis of tubercular meningitis. J Trop Pediatr. 2000 Dec;46(6):378-9.

9. Watt G, Zaraspe G, Bautista S, Laughlin LW: Rapid diagnosis of tuberculous meningitis by using an enzyme linked immunosorbent assay to detect mycobacterial antigen and antibody in cerebrospinal fluid. J Infect Dis. 1988 Oct;158(4):681-6.

10. Katti MK: Assessment of antibody responses to antigens of Mycobacterium tuberculosis and Cysticercus cellulosae in cerebrospinal fluid of chronic meningitis patients for definitive diagnosis as TBM/NCC by passive hemagglutination and immunoblot assays. FEMS Immunol Med Microbiol. 2002 Mar 25;33(1):57-61.

11. Mathai A, Radhakrishnan VV, Sarada C, George SM: Detection of heat stable mycobacterial antigen in cerebrospinal fluid by Dot-Immunobinding assay. Neurol India. 2003 Mar;51(1):52-4.

12. Krambovitis E, McIllmurray MB, Lock PE, Hendrickse W, Holzel H: Rapid diagnosis of tuberculous meningitis by latex particle agglutination. Lancet. 1984 Dec 1;2(8414):1229-31.

13. Kashyap RS, Dobos KM, Belisle JT, Purohit HJ, Chandak NH, Taori GM, Daginawala HF: Demonstration of components of antigen 85 complex in CSF of Tuberculous meningitis patients. Clin Diagn Lab Immunol. 2005 Jun;12(6):752-8.

14. Rana SV, Chacko F, Lal V, Arora SK, Parbhakar S, Sharma SK, Singh K. To compare CSF adenosine deaminase levels and CSF-PCR for tuberculous meningitis. Clin Neurol Neurosurg. 2010 Jun;112(5):424-30. 
15. Baro M, Acevedo L, Lagos ME: Usefulness of adenosine deaminase determination in cerebrospinal fluid for the diagnosis of meningeal tuberculosis; 4 years experience at a public hospital. Rev Med Chill. 1996 Mar;124(3):319-26.

16. Pettersson T, Klockars M, Weber TH: Diagnostic value of cerebrospinal fluid adenosine deaminase determination. Scand J Infect Dis. 1991 Jan;23(1):97100 .

17. Blake J, Berman P: The use of adenosine deaminase assays in the diagnosis of tuberculosis. S Afr Med J. 1982 Jul 3;62(1):19-21.

18. Mishra OP, Loiwal V, Ali Z, Nath G, Chandra L, Das BK. Cerebrospinal fluid adenosine deaminase activity and C-reactive protein in tuberculous and partially treated
Research Article

bacterial meningitis. Indian Pediatr. 1995 Aug;32(8):886-9.

19. Rana SV, Singhal RK, Singh K, Kumar L. Adenosine Deaminase levels in Cerebrospinal fluid as a diagnostic test for Tuberculous Meningitis in children. Indian J Clin Biochem. 2004 Jul;19(2):5-9.

20. Baheti R, Laddha P, Gehlot RS. CSF-Adenosine Deaminase (ADA) Activity in Various Types of Meningitis. J Ind Acad Clin Med. 2001 Dec;2(4):285-7.

21. Cho BH, Kim BC, Yoon GJ, Choi SM, Chang J, Lee $\mathrm{SH}$, et al. Adenosine deaminase activity in cerebrospinal fluid and serum for the diagnosis of tuberculous meningitis. Clin Neurol Neurosurg. 2013 Sep;115(9):1831-6.

\section{How to cite this article?}

Shrivastava J, Agrawal A, Patel D. Simultaneous estimation of serum and cerebrospinal fluid adenosine deaminase level to differentiate tuberculous and non-tuberculous meningitis. Int $J$ Med Res Rev 2014;2(5):414- 419. doi:10.17511/ijmrr.2014.i05.03 